\title{
Real-time Fully Automated Internal Layer Segmentation of Human Retina in Optical Coherence Tomography Images
}

\author{
Youngmin Han, Naresh Kumar Ravichandran, Pilun Kim, Mansik Jeon, Jeehyun Kim
}

\begin{abstract}
In the field of ophthalmology, optical coherence tomography (OCT) has proven to be a powerful imaging technique when it comes to diagnosing various eye-related diseases. This research article introduces a real-time automatic retinal layer segmentation algorithm based on intensity variation in the OCT images. The built algorithm is capable of detecting internal retinal layers like the internal limiting membrane (ILM), the retinal pigment epithelium (RPE) and the retinal nerve fiber layer (RNFL) with micrometer level precision, the algorithm uses openMP for parallelized computation for real-time visualization of the segmented retinal layers. The total execution time of the algorithm was evaluated using various image sizes and compared with the OCT frame rate to demonstrate the efficiency of real-time segmentation.
\end{abstract}

Keywords: Layer Segmentation, Optical Coherence Tomography, Real-time, Retina.

\section{INTRODUCTION}

W hen it comes to early diagnosis of diseases in the field of ophthalmology, optical coherence tomography (OCT) is seen as a well-established and reliable imaging technique. The advent of OCT and its capabilities to get 3-dimensional and cross-sectional images of biological tissues has made it possible for ophthalmologists and researchers to study the retinal layers non-destructively [1-4]. OCT has been used for many early disease diagnoses, and one such area where OCT is seen as a fascinating imaging tool is for diagnosis of glaucoma. When the onset of glaucoma disease becomes severe over time, the intraocular pressure (IOP) is increased within the eye, and this leads to a reduction in the rate of aqueous secretion within the eye. Furthermore, the internal

Revised Manuscript Received on February 10, 2020.

* Correspondence Author

Youngmin Han, Department of Nuclear Energy Convergence, Kyungil University, Gyeongsan-si, Gyeongbuk, Republic of Korea. Email: hanym116@gmail.com.

Naresh Kumar Ravichandran, School of Electronics Engineering, College of IT Engineering, Kyungpook National University, 80 Daehak-ro, Bukgu, Daegu 41566, Republic of Korea. Email: nareshr.9169@gmail.com.

Pilun Kim, Institute of Biomedical Engineering Research, Kyungpook National University, 680, Gukchaebosang-ro, Jung-gu, Daegu 41944, Republic of Korea. Email: pukim@knu.ac.kr.

Mansik Jeon, School of Electronics Engineering, College of IT Engineering, Kyungpook National University, 80 Daehak-ro, Bukgu, Daegu 41566, Republic of Korea. Email: msjeon@knu.ac.kr.

Jeehyun Kim, School of Electronics Engineering, College of IT Engineering, Kyungpook National University, 80 Daehak-ro, Bukgu, Daegu 41566, Republic of Korea. Email: jeehk@knu.ac.kr.

(C) The Authors. Published by Blue Eyes Intelligence Engineering and Sciences Publication (BEIESP). This is an open access article under the CC BY-NC-ND license (http://creativecommons.org/licenses/by-nc-nd/4.0/) tissues and nerve fibres of retina get damaged, leading to the destruction of internal retinal structures. Additionally, the thickness of the retinal nerve fiber layer (RNFL) is also decreased. In conventional imaging and diagnosis techniques in ophthalmology, wither requires sectioning or only the topological structure of the retina can be visualized. With the use of OCT imaging, it becomes possible to diagnose the disease by analyzing the internal morphology of the retinal layers. When it comes to glaucoma and other ophthalmological disease diagnoses, the measurement of the thickness of the retina and the RNFL becomes a key factor [5]. In general, the internal retinal layers in the OCT images have been segmented manually by the ophthalmologist to precisely evaluate the condition of the eye to estimate the progress or severity of. Among the various layers in retina images, the retinal pigment epithelium (RPE) and the internal limiting membrane (ILM) are the most recognized internal layers in the OCT images of the retina. RPE is the layer that lies between the choroid and visual cells of the retina. However, RPE and outer tips of photoreceptors are the most highly-scattering layer in OCT images [6]. Hence, these two layers are usually appearing as a single line, and it is recognized as an RPE complex [7]. Therefore, the RPE complex also reflects the state of the RPE layer. ILM is also an important structure, and it is the layer found between the retina and the vitreous section of the eye [8]. In OCT images, the retinal thickness is measured by calculating the total distance between the ILM and RPE layers. Calculation of these layer thickness is crucial for ophthalmology disease diagnosis and also serves as an important parameter making the treatment planning. The total measurement of this thickness directly corresponds to the RNFL thickness, and it is used to evaluate the progress of glaucoma in patients. Using OCT images, it also becomes feasible to study the inner most layers in the retinal structure. In the past, several image analysis methods have been proposed for retinal layer segmentation [9, 10]. However, the 3-dimensional image acquisition has always been a highly time-consuming process. Also, the involuntary physical movement of the patients makes it difficult for acquiring a real-time 3-dimensional OCT image with automatically segmented internal layers. A high real-time image acquisition rate is a crucial factor in medical imaging modalities that are used for diagnosis and treatments like surgeries. In recent years, automated segmentation of retinal layers to identify the retinal structures using video-rate is widely used. However, this method is most suitable to only identify the ILM layer.

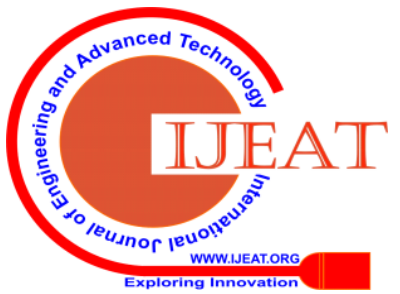


This makes a real-time overall thickness measurement and segmentation of retinal layers a growing research field that has fascinated the researchers worldwide.

In this research article, we have introduced a real-time automatic retinal layer segmentation algorithm based on intensity variation in the OCT images. The introduced algorithm is fast enough for real-time 3-dimensinal OCT image acquisition with segmented internal retinal layers. The proposed algorithm can effectively identify the RPE, ILM, and RNFL layers, along with their total layer thickness measurement analysis.

\section{METHODS}

The steps included in the proposed algorithm is shown graphically in Fig. 1(a).

The OCT data set (cross-sectional image, i.e. B-scan image) consists of $400 \mathrm{~A}$-scans (depth scans). In the acquired cross-sectional OCT images, the RPE and ILM layers are detected first, by using the information obtained from the detected layers, the images are aligned based on the RPE

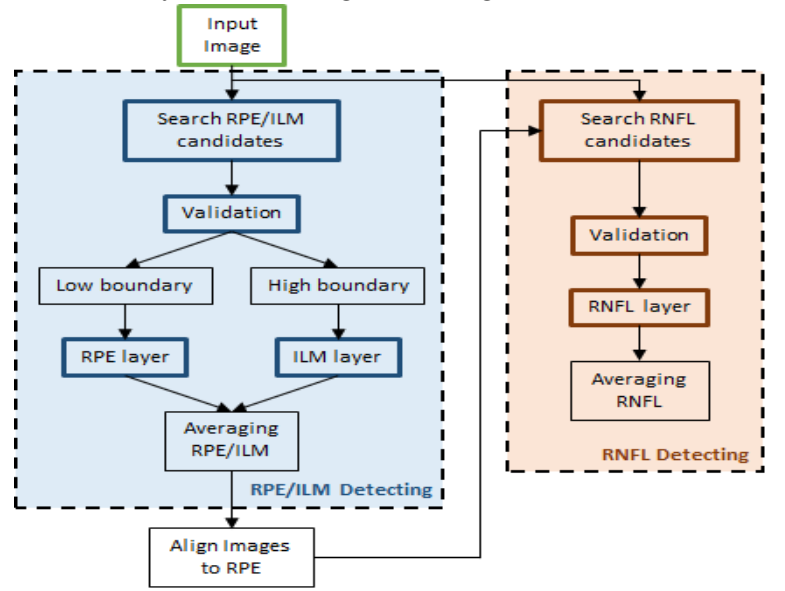

(a)

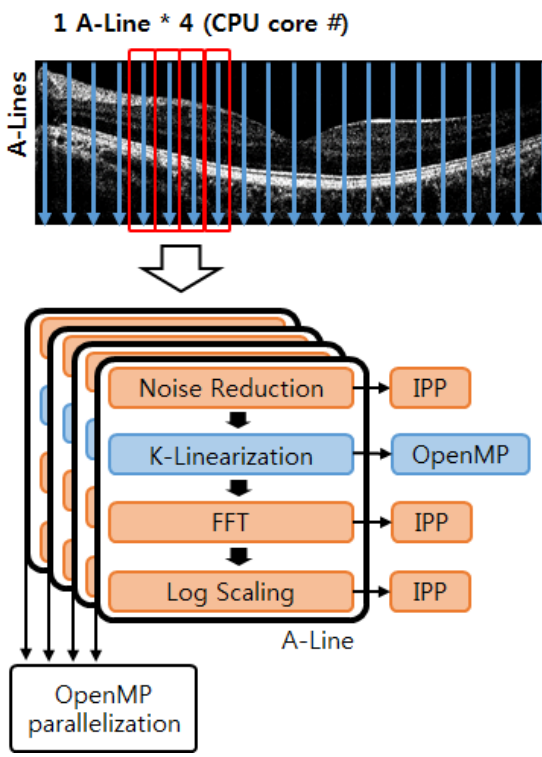

(b)

Fig. 1.Schematic of the layer detection algorithm and image acquisition protocol. (a) Flow diagram explaining the process involved in layer detection algorithm (b) Image acquisition process using OpenMP and IPP (Intel Integrated Performance primitives) layer. The RNFL layer is detected between the positions of RPE and ILM layers within the OCT retinal image.

\section{A. Data acquisition}

All OCT images were acquired by a commercially available OCT system, The Retina FOI 2 (Oz-tec Co., Ltd., Daegu, Republic of Korea). The center wavelength of the laser source used in the OCT system is $840 \mathrm{~nm}$, and the full width half maximum bandwidth is $50 \mathrm{~nm}$ for the broadband light source. The scanning rate of the line-scan camera is 50 $\mathrm{kHz}$ per second. The axial resolution of the system is $5.2 \mu \mathrm{m}$ in tissue (refractive index $=1.38$ ). The images were acquired at 20 frames for $27 \mathrm{~s}$, and the overall image size acquired by the OCT system is 756 (transverse) x 500 (depth) x 540 (frame). The detailed process involved in the OCT image acquisition is shown in Fig. 1(b). The process of noise reduction, fast Fourier transform (FFT), and log scaling steps are supported by Intel Integrated Performance primitives (Intel IPP), and the k-linearization is supported by OpenMP. The computation of every A-scan is also parallelized by OpenMP decided according to the number of the available $\mathrm{CPU}$ cores. The total data acquisition time for one cross-sectional OCT image is $11 \mathrm{~ms}$.

\section{B. RPE and ILM layer detection}

Prior to the detection of the RPE and ILM layers, the pre-determined mask (as shown in Fig. 2(b)) is modelled on to the edge of the layer in the OCT image, as shown in Fig. 2(a). The $\mathrm{S}(\mathrm{x}, \mathrm{y})$, which represents the similarity value is calculated by (1).

$$
\mathrm{S}(\mathrm{x}, \mathrm{y})=\sum_{\mathrm{i}=-1}^{1} \sum_{i=-5}^{14} w_{i, j} \Delta f(x+i, y+j)
$$

Here $f(x, y)$ is the intensity at the location $(x, y)$ and $W_{i, j}$ is the function of the mask $\mathrm{W}$ at the location $(\mathrm{x}, \mathrm{y})$ which is defined as in the (2).

$$
w_{i, j} \Delta f(x, y)=\left\{\begin{array}{l}
1 \text { if } j>0 \text { and } f(x, y)<T h r 1 \\
1 \text { if } j \leq 0 \text { and } f(x, y) \geq T h r 1 \\
0 \text { otherwise }
\end{array}\right.
$$

In the above (2), the $\mathrm{S}(\mathrm{x}, \mathrm{y})$ is the value that represents the similarity of intensity pixels (at the edge of the layer) compared with the mask at the respective locations (x,y). After execution of the image mask $\mathrm{W}$, the highest two values obtained for $\mathrm{S}$, i.e., $\mathrm{S}\left(\mathrm{x}, \mathrm{y}_{1}\right)$ and $\mathrm{S}\left(\mathrm{x}, \mathrm{y}_{2}\right)$, are detected along the depth direction of the A-Scans (depth scans) is shown in Fig. 3(b), Fig. 4(a). These two locations ( $\left.x, y_{1}\right),\left(x, y_{2}\right)$ are detected for the location of ILM and RPE layers in a B-Scan image. Following this, the validation step is performed for evaluating whether the detected locations $\left(\mathrm{x}, \mathrm{y}_{1}\right)$ and $\left(\mathrm{x}, \mathrm{y}_{2}\right)$ are not erroneous.

For the validation, $\mathrm{I}_{\mathrm{avg}}$, the average of the intensity of size $11 \mathrm{x} 11$ pixels, at the location $\left(\mathrm{x}, \mathrm{y}_{1}\right)$ and $\left(\mathrm{x}, \mathrm{y}_{2}\right)$ is evaluated. This is shown in (3). 


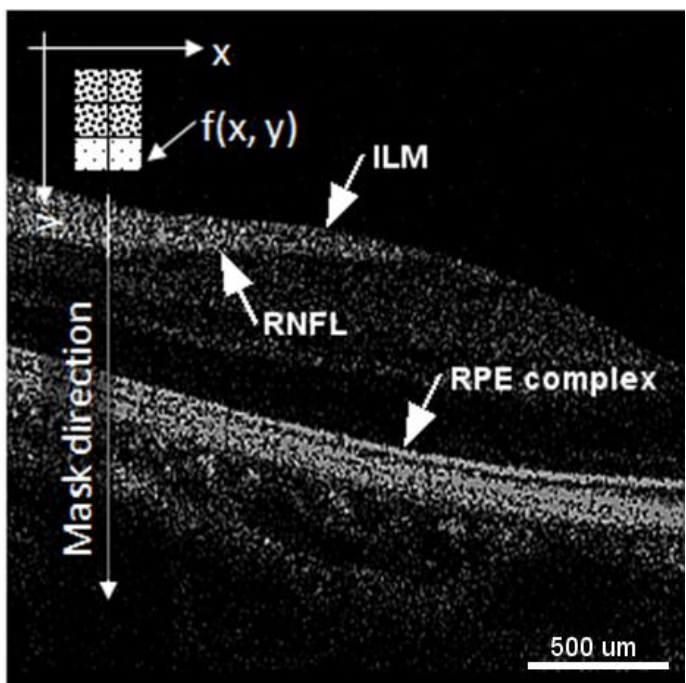

(a)

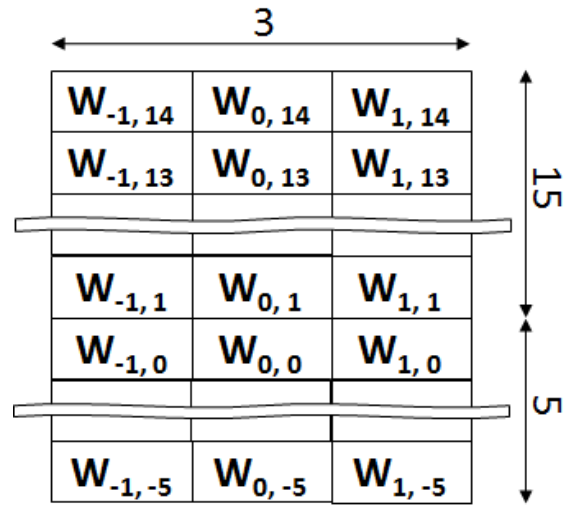

(b)

Fig. 2. Applying mask in OCT image before layer segmentation. (a) Masking process in the sample image, (b) the example of the mask $\mathrm{W}$

$$
I_{\text {avg }}(x, y)=\sum_{i=-5}^{5} \sum_{j=-5}^{5} f\left(x+i_{i} y+j\right) /(11 \times 11)
$$

Since the intensity layer, the OCT image is brighter than background pixel intensity $\mathrm{I}_{\mathrm{bg}}$, $\mathrm{I}_{\text {avg }}$ must be higher than $\mathrm{I}_{\mathrm{bg}}$. If $I_{\text {avg }}(x, y)$ is smaller than $I_{b g}$, the location $(x, y)$ is ignored as this is erroneous. An example of this scenario is shown in the bottom left inset image in Fig. 3(c).

$$
(\mathrm{x}, \mathrm{y})=\left\{\begin{array}{l}
(x, y) \text { if } I_{\text {avg }}(x, y) \geq I_{\text {bg }} \\
(x, 0) \text { otherwise }
\end{array}\right.
$$

It is known that the position of the ILM layer in retina precedes the RPE layer. Therefore, $\mathrm{y}_{\mathrm{ILM}-\mathrm{C}}(\mathrm{x})$ and $\mathrm{y}_{\mathrm{RPE}-\mathrm{C}}(\mathrm{x})$, which represent the location of the ILM and RPE layer at a B-scan, are defined by (5).

$$
\begin{aligned}
& y_{L L M-c}(\mathrm{x})=\min \left(y_{1}, y_{2}\right) \text { at } x \text { th } B-\operatorname{scan} \\
& y_{R P E-c}(\mathrm{x})=\max \left(y_{1}, y_{2}\right) \text { at } x \text { th } B-\operatorname{scan}
\end{aligned}
$$

In the next step, the $\mathrm{y}_{\mathrm{ILM}}(\mathrm{x})$ and $\mathrm{y}_{\mathrm{RPE}}(\mathrm{x})$, which represents the layer location of ILM and RPE at $\mathrm{x}$ a B-scan, are obtained by smoothening the $\mathrm{y}_{\mathrm{ILM}-\mathrm{C}}(\mathrm{x})$ and $\mathrm{y}_{\mathrm{RPE}-\mathrm{C}}(\mathrm{x})$ which is obtained from (5). The calculation for this step is shown in (6).

$$
y_{k}(x)= \begin{cases}y_{k}(x-1)+1 & \text { if } y_{k-c}(x)>y_{k}(x-1) \\ y_{k}(x-1)-1 & \text { if } y_{k-c}(x)<y_{k}(x-1) \\ y_{k}(x-1) & \text { if } y_{k-c}(x)=y_{k}(x-1) \\ y_{k}(x-1) & \text { if } y_{k-c}(x)=0\end{cases}
$$

Here k represents the ILM and RPE or RNFL for section 2.3.

After the smoothening, the original image is flattened based on the RPE layer so that the RPE layer appears a constant line; this is shown in Fig. 3(d).

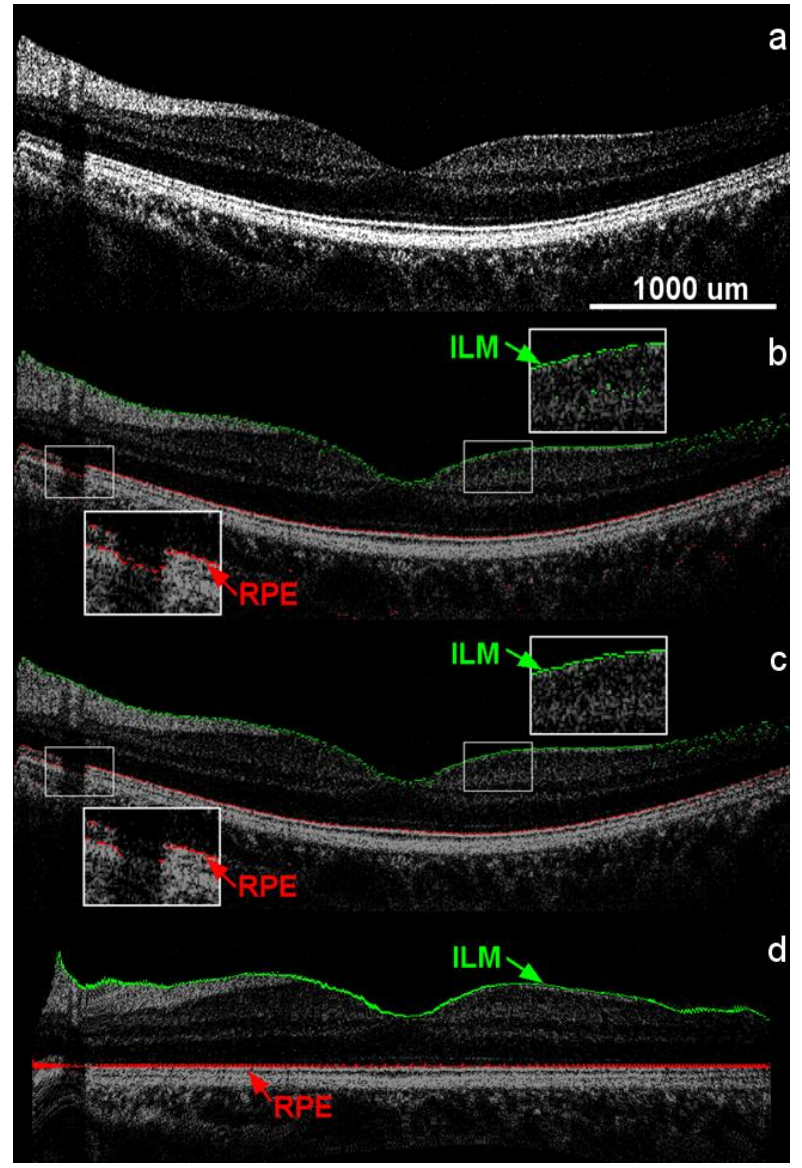

Fig. 3. OCT image after each step involved in the layer detection algorithm. (a) Original image, (b) Indication of two greatest values of S, i.e., ILM (green) and RPE (red), (c) Evaluation of the two $S$ values and smoothening, (d) Flattened OCT image after detection of ILM and RPE layers.

\section{RNFL layer detection}

It is a well understood concept that the RNFL layer is located between the ILM and RPE layers. Therefore, it is possible to specify a region of interest to detect the RNFL layer within the retina. The pixel intensities (within) RNFL layer is not greater than ILM or RPE layers. Therefore, it is necessary to compare the pixel intensity of the image, and the $\mathrm{I}_{\text {sum, i.e., the }}$ sum of the intensity of size $5 \times 5$ pixels. This gives the region of interest for the RNFL location $(x, y)$, which lies between ILM and RPE layers.

$$
l_{\text {sum }}(x, y)=\sum_{i=-2}^{2} \sum_{j=-2}^{2} f(x+i, y+j)
$$

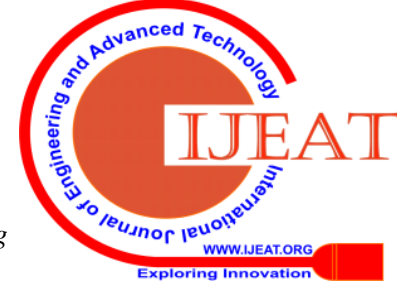


From the above (7), the intensities $\mathrm{I}_{\text {sum }}$ between ILM and RNFL layers are greater than that of RNFL, and between RPE layers are shown as an inset image in Fig. 4(b). However, the RNFL layer does not appear at some of the B-Scan regions. In this case, the RNFL layer is defined with a threshold value (Thr). The algorithm is designed in such a way that if the obtained value of $\mathrm{I}_{\text {sum }}(\mathrm{x}, \mathrm{y})$ is lower than Thr, it is assumed that $(\mathrm{x}, \mathrm{y})$ is below the RNFL layer. Therefore, $\mathrm{y}_{\mathrm{RNFL}-\mathrm{C}}(\mathrm{x})$, that is, the location of the RNFL layer in a B-scan, is given as the following (8).

$$
\begin{aligned}
& y_{\text {RNFL }-c}(x)=\min \left(y \mid I_{\text {sum }}(x, y)<I_{\text {sum }}\left(x_{*} y_{I L M}(x)\right) *\right. \\
& \left.k_{\text {int }} \text { or } I_{\text {sum }}(x, y)<T h r 2\right)
\end{aligned}
$$

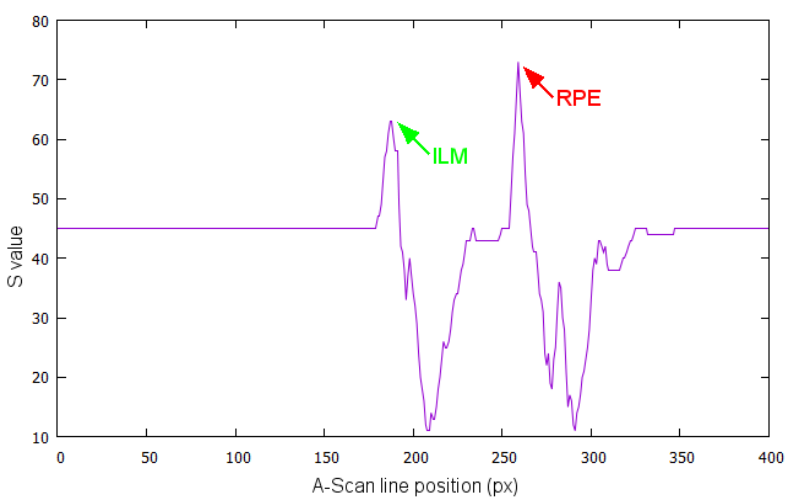

(a)

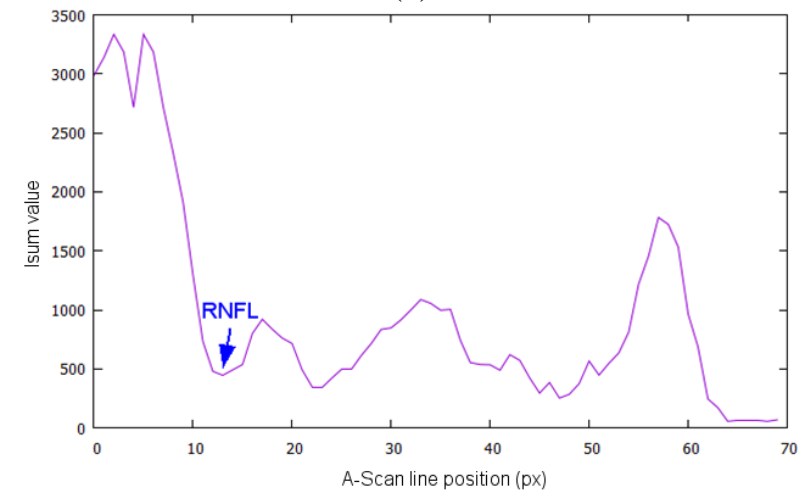

(b)

Fig. 4. A-scan analysis of the OCT image to determine ILM and RPE layers. (a) Example of detected S values, i.e., ILM and RPE along the

A-Scan line, the highest detected intensities in A-scan corresponds to the ILM and RPE layers, respectively.

(b) Example of the averaged Isum graph, and the first deep peak corresponds to the RNFL layer.

In the above (8), the $\mathrm{k}_{\mathrm{int}}$ represents the intensity difference between the above and below the RNFL layer coefficient (Fig. 5(a). Here, the Thr is set to 1250 , and the $\mathrm{k}_{\text {int }}$ is set to 0.5 . The same validation process utilized in (3) and (4) in the aforementioned section 2.2 is applied to $\mathrm{y}_{\text {RNFL-C }}(\mathrm{x})$; this is shown in Fig. 5(b). The obtained $\mathrm{y}_{\mathrm{RNFL}-\mathrm{C}}(\mathrm{x})$ is smoothened in the same procedure as explained in (6), and the final $\mathrm{y}_{\mathrm{RNFL}}(\mathrm{x})$ position is obtained; this is shown in Fig. 5(c).

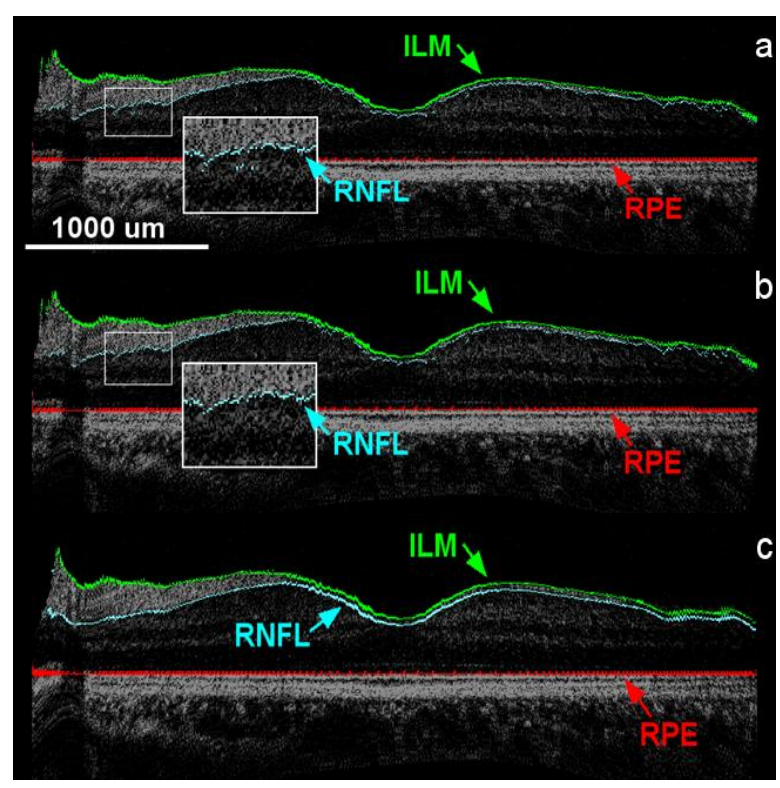

Fig. 5. RNFL layer detention in cross-sectional OCT images. (a) Candidates of RNFL layer (blue). (b) Validation of the RNFL candidates (blue). (c) the final smoothened RNFL layer blue below the ILM layer (green).

\section{RESULTS}

\section{A. Layer segmentation performed in the macula region}

The measurement of the macula is performed with the OCT images obtained from a 3-dimensional scan, and the dataset is of 800 (depth) x 600 (transverse) pixels, and 300 frames were used for the layer segmentation process. The 200 pixels (transverse) and 100 frames of the original dataset were neglected due to its low pixel intensity. The projection image of the obtained 3-dimensionla scan along with, 5 cross-section images at different positions in the projection image (marked from 1-5) is shown in Fig. 6 (b)-(f) with the automatically detected and segmented internal layers like RPE, ILM and RNFL It is to be noted that only a negligible amount of errors have occurred during the automatic layer segmentation process. The rate of false segmentation (error occurrence) during the detection of RPE, ILM, and RNFL layers was calculated manually along the B-scans for every 10 images. It is observed that the gap between the real layer and erroneously detected layer is no bigger than $10 \%$ of the average retina thickness $(244 \pm 63 \mu \mathrm{m})$. During the error evaluation, $97.4 \%$ of RPE segmentation, 99.9\% of ILM segmentation, and $97.3 \%$ of RNFL segmentation were detected correctly.

The Fig. 7 shows the retina thickness and RNFL thickness of the whole scan area on $2 \mathrm{D}$ position maps. The retina thickness is calculated by measuring the distance between the RPE layer and the ILM layer, and the RNFL thickness corresponds to the distance between the RNFL layer and the ILM layer. The real thickness of the layer is indicated by the color bar.

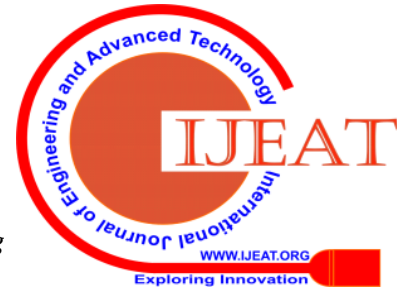


According to the dataset of layer position and its thickness, the retina 3D structure is shown in Fig 8. This proves that the proposed algorithm makes it possible to observe each layer individually in volumetric data.
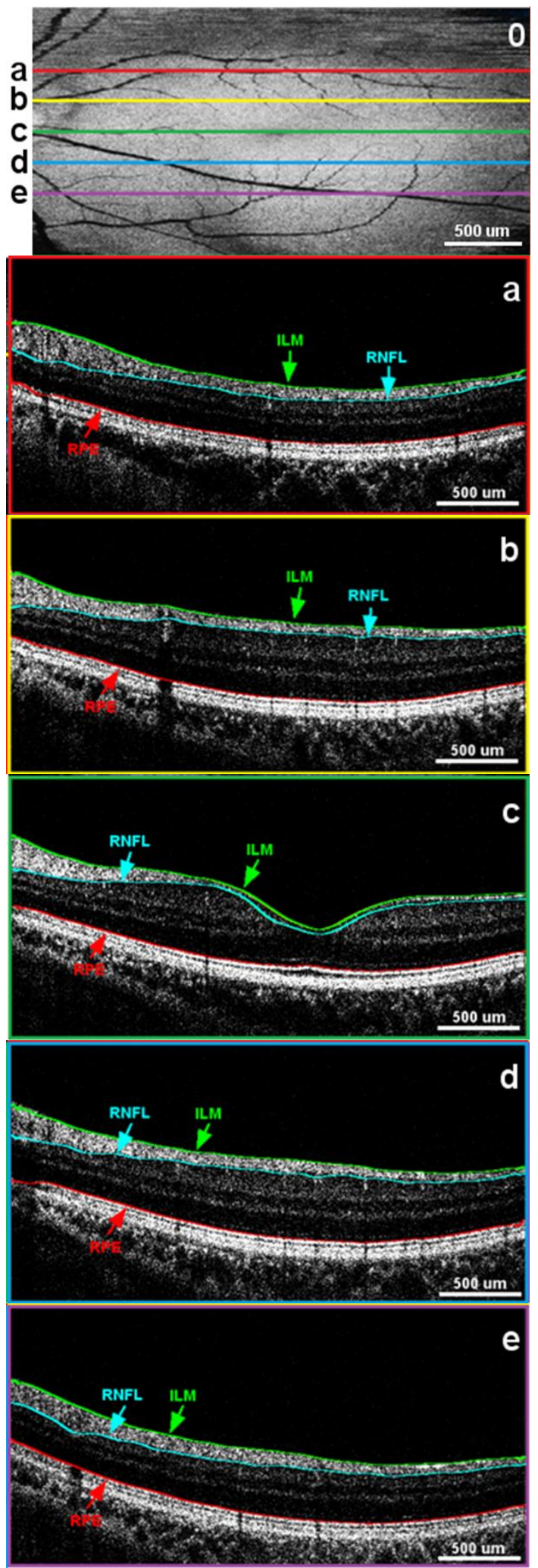

Fig. 6. Projection image and 5 cross-section images with RPE, ILM, and RNFL layers. The cross-sectional positions are represented in the projection image as lines 1-5.

Retrieval Number: C5632029320/2020@BEIESP

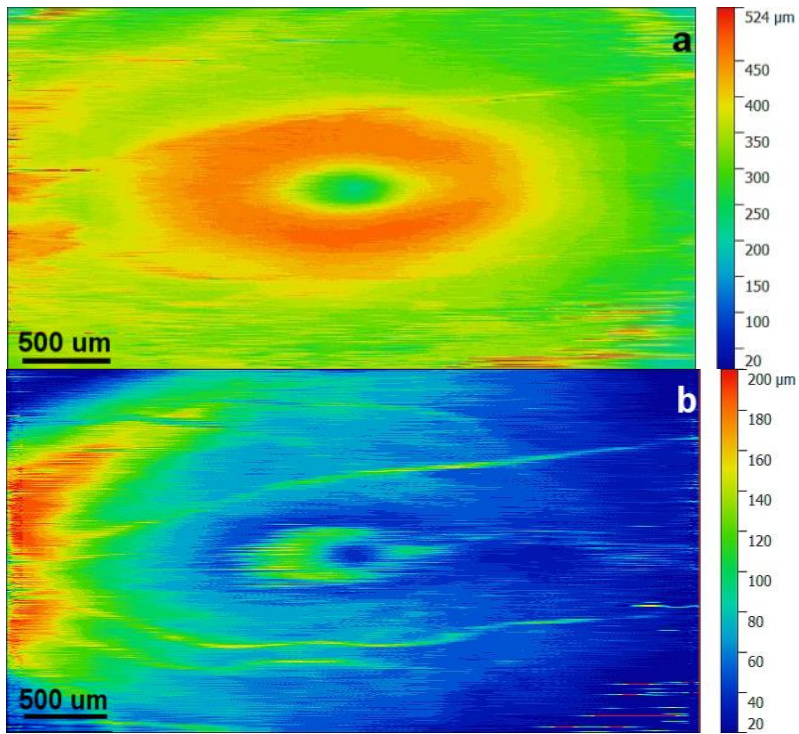

Fig. 7.Measured thickness in the retina. Total Retina thickness (left) and RNFL thickness (right). The values of the color bar show the measured real thickness values

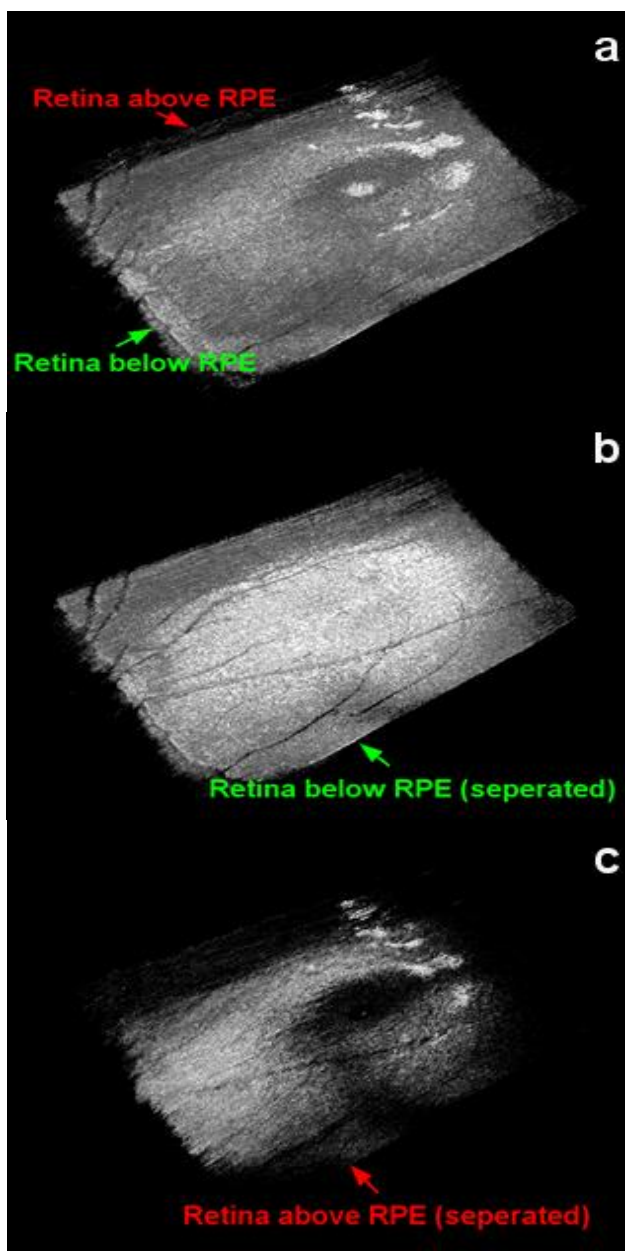

Fig. 8. 3D images of the retina after layer segmentation. Entire retina structure (left), Retina below RPE layer structure (center), Retina above RPE layer structure (right).

Published By:

Blue Eyes Intelligence Engineering

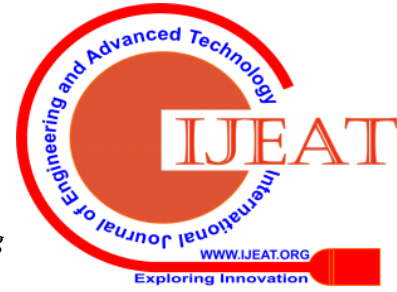




\section{B. Execution time}

The average execution time of the proposed segmentation method is evaluated on an image-set $(\mathrm{M}, \mathrm{N})$ with 390 frames using Intel i7-4790, 3.6 GHz with 32GB as the main memory. Each image-set $(\mathrm{M}, \mathrm{N})$ consists of a different number of pixels $\mathrm{M}$ (depth) $\mathrm{x} \mathrm{N}$ (transverse). All images were resized $(800,800)$ from the original image-set. The execution time for the image-set $(800,800)$ was $48 \mu$ s per frame which is consisted of $29 \mu \mathrm{s}$ for RPE/ILM segmentation, $18 \mu \mathrm{s}$ for RNFL segmentation, and $1 \mu$ s for image alignment. Furthermore, it was noted that, if the image size is reduced, the required time for the segmentation is also reduced. It takes about is $16 \mu$ s per frame when the image size $(\mathrm{M} \mathrm{x} \mathrm{N})$ is 160000 pixels $(M=400, N=400)$.

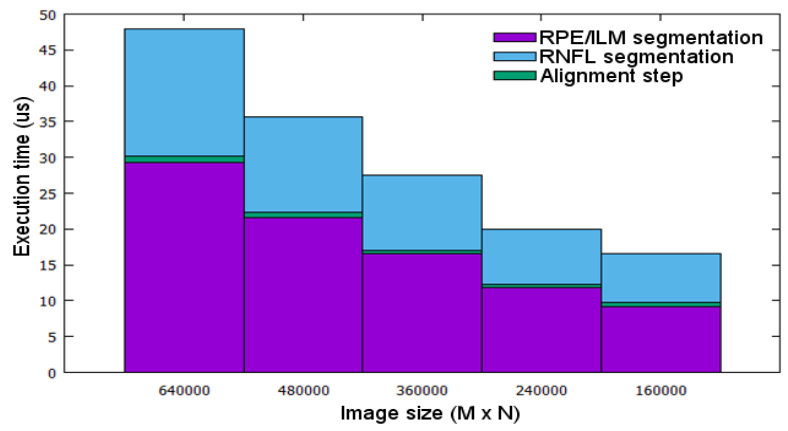

(a)

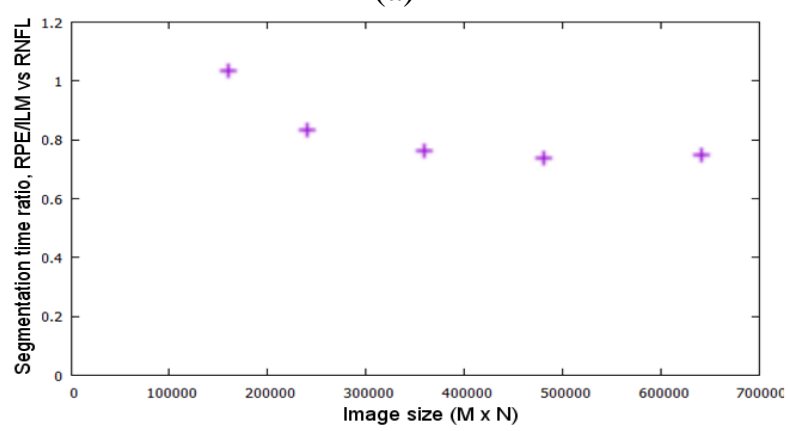

(b)

Fig. 9. Evaluation of overall execution time for the proposed algorithm. (a) The execution time per each step according to the image size (b) The execution time ratio, RPE/ILM segmentation vs RNFL segmentation

The total execution time, according to image size, is plotted as a bar graph and shown in Fig. 9(a). The highest time-consuming part of the proposed algorithm is the detection of RPE/ILM segmentation, which takes more than 55 percent of the total execution time. The RNFL segmentation step takes $37 \sim 41 \%$ of the total execution time, and the alignment step takes less than $3 \%$ of the total execution time. No dependency was observed between the execution time ratio of each step and $\mathrm{M} x \mathrm{~N}$ size. When the image size is bigger than 360,000 pixels, it is constant. However, it increases when the image size is smaller than 360,000 pixels. The total execution time ratio for different image sizes is shown in Fig. 9(b).

\section{DISCUSSION}

The proposed segmentation method has been proven to be effective in internal retinal layer identification. From the obtained result evaluations, the ILM segmentation shows less than a $0.1 \%$ error ratio, and the RPE/RNFL shows less than a $3 \%$ error ratio. Most of the erroneous case has occurred near blood vessel regions, and this is shown in Fig. 10. It is possible to observe the blood vessel in the projection images, and the RNFL layer in the cross-section image at the same location is found to be slightly thicker than the detected RNFL layer in other locations in the macula. Similarly, the RPE layer near the blood vessel regions is nearly absent. This could be due to the presence of the layer is changes rapidly near these regions. Hence the averaging method does not show the exact position of the layer. For these reasons, RNFL and RPE layers are not detected perfectly near the blood vessel regions. However, the ILM layer is not affected by the blood vessel, and thus it shows a very high ratio of exact segmentation. The segmentation errors mentioned above can also occur at discontinuous layers due to two reasons, i.e., disease occurrence or due to low pixel intensities. In the case of low pixel intensities, the layer segmentation results shown in section 3.1 are performed with 600 (transverse) pixels.

The OCT system used in this study produces 20 OCT images per second. Thus, the segmentation process is to be completed within $50 \mu$ s to achieve a high video-rate image process. From the obtained results, it is evident that the proposed algorithm is fast enough to produce a video-rate segmentation process with an OCT image of size 800 x 800 pixels. If the size of the images is reduced, a shorter segmentation time and a high OCT frame rate can be achieved. The execution time decreases with a reduction in image size. In this research, OpenMP is used for the parallelization process. Therefore, some part of the algorithm is parallelized and shows very high-speed performance, even with a high amount of data (larger image size). However, the other parts which are hard to be parallelized cannot benefit from the OpenMP method. These parts take a relatively longer time to execute. Therefore, the total execution time per image size is long with smaller image sizes. If this algorithm is executed with an improved parallelizing method such as graphic processing units, the execution time can be reduced even further.

\section{CONCLUSION}

A new real-time retina segmentation algorithm for ILM, RPE, and RNFL layers method based on intensity variation in OCT images has been shown. From the obtained result, the overall performance of the proposed algorithm is demonstrated for three different retinal layer segmentation without pre-processing. The proposed method is a fully automated mechanism requiring no assistance from the user. In our experiments, the data, which consisted of 800 (depth) $\mathrm{x}$ 800 (transverse) pixels, took $48 \mu$ s per frame to segment all three retinal layers. The proposed algorithm can aid researchers and ophthalmologists to study and diagnose the physical state of the different internal layers of the retina and to check for continuity of retinal layers to assess for damage in internal retinal layers. 


\section{ACKNOWLEDGMENT}

This research was supported by Kyungpook National University Development Project Research Fund, 2018.

\section{REFERENCES}

1. I. Voo, E. C. Mavrofrides, and C. A. Puliafito, "Clinical applications of optical coherence tomography for the diagnosis and management of macular diseases," Ophthalmology Clinics of North America, vol. 17, no. 1, pp. 21-31, 2004

2. S. G. Schuman, A. F. Koreishi, S. Farsiu, S.-h. Jung, J. A. Izatt, and C. A. Toth, "Photoreceptor layer thinning over drusen in eyes with age-related macular degeneration imaged in vivo with spectral-domain optical coherence tomography," Ophthalmology, vol. 116, no. 3, pp. 488-496. e2, 2009.

3. Y. Zhao, Z. Chen, C. Saxer, S. Xiang, J. F. de Boer, and J. S. Nelson, "Phase-resolved optical coherence tomography and optical Doppler tomography for imaging blood flow in human skin with fast scanning speed and high velocity sensitivity," Optics letters, vol. 25, no. 2, pp. 114-116, 2000.

4. M. F. Shirazi, R. E. Wijesinghe, N. K. Ravichandran, P. Kim, M. Jeon, and J. Kim, "Dual-path handheld system for cornea and retina imaging using optical coherence tomography," Optical Review, vol. 24, no. 2, pp. 219-225, 2017.

5. J. L. Wheat, N. V. Rangaswamy, and R. S. Harwerth, "Correlating RNFL thickness by OCT with perimetric sensitivity in glaucoma patients," Journal of glaucoma, vol. 21, no. 2, p. 95, 2012.

6. Y. Ikuno et al., "Reproducibility of retinal and choroidal thickness measurements in enhanced depth imaging and high-penetration optical coherence tomography," Investigative ophthalmology \& visual science, vol. 52, no. 8, pp. 5536-5540, 2011.

7. J. J. Weiter and R. Zuckerman, "The influence of the photoreceptor-RPE complex on the inner retina: an explanation for the beneficial effects of photocoagulation," Ophthalmology, vol. 87, no. 11, pp. 1133-1139, 1980 .

8. A. S. Reis, N. O'Leary, M. J. Stanfield, L. M. Shuba, M. T. Nicolela, and B. C. Chauhan, "Laminar displacement and prelaminar tissue thickness change after glaucoma surgery imaged with optical coherence tomography," Investigative ophthalmology \& visual science, vol. 53, no. 9, pp. 5819-5826, 2012.

9. D. C. Fernández, H. M. Salinas, and C. A. Puliafito, "Automated detection of retinal layer structures on optical coherence tomography images," Optics express, vol. 13, no. 25, pp. 10200-10216, 2005.

10. S. J. Chiu, X. T. Li, P. Nicholas, C. A. Toth, J. A. Izatt, and S. Farsiu, "Automatic segmentation of seven retinal layers in SDOCT images congruent with expert manual segmentation," Optics express, vol. 18, no. 18, pp. 19413-19428, 2010.

\section{AUTHORS PROFILE}

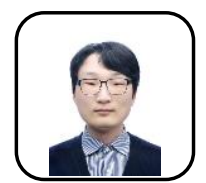

Youngmin Han, received his B.E degree in mechanica engineering from Tohoku University, Sendai, Japan in 2012. He completed his M.Sc. in power engineering from Technical University of Munich, Munich, Germany. His research interests are in the development of advanced materials, including tungsten metals and molten salts, for nuclear fusion reactors using optical techniques and image processing.

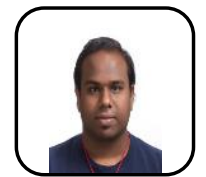

Naresh kumar Ravichandran, received his B.E degree in electronics and communication engineering from St. Peter's University, Tamil Nadu, India. He completed his M.S. in electronics engineering from Kyungpook National University, Daegu, Republic of Korea and currently, he is a Ph.D. researcher in the Electronics Engineering Department of Kyungpook National University, since 2016. His research interest is in the developing and optimizing novel biological imaging techniques with possible applications in agronomical studies, entomological studies, industrial applications, and for dental applications.

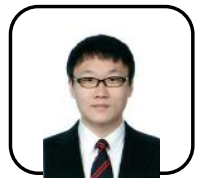

Pilun Kim, is received his Ph.D Degree in 2011 from the Department of Medical and Biological Engineering at the Kyungpook National University. And he is currently a Researching Visiting Professor in Institute of Biomedical Engineering at Kyungpook National University. He is interested in translating new technologies from the research field to the application field such as clinic and industrial and making its Productization. Main interests are Biomedical device development, Optical Coherence Tomography (OCT) and Digital Image Processing.

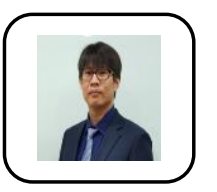

Mansik Jeon received the Ph.D. degree in electronics engineering from Kyungpook National University, Daegu, South Korea, in 2011. He is currently as Assistant Professor with the School of Electronics Engineering, Kyungpook National University. His research interests are in the development of nonionizing and noninvasive novel biomedical imaging techniques, including photoacoustic tomography, photoacoustic microscopy, optical coherence tomography, ultrasonic imaging, handheld scanner, and their clinical applications.

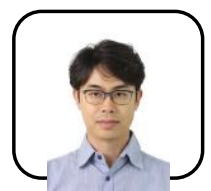

Jeehyun Kim received the Ph.D. degree in biomedical engineering from the University of Texas at Austin, USA, in 2004. He was a Post-Doctoral Researcher with the Beckman Laser Institute, University of California at Irvine. He is currently a Full Professor with Kyungpook National University, Daegu, South Korea. His research interest is in biomedical imaging and sensing, neuroscience studies using multiphoton microscopy, photo-acoustic imaging, and other nove applications of sensors. 